\title{
SALUD MENTAL, CIUDADES Y URBANISMO: 1. SOBRE PSICOSOMÁTICA Y PSICODINÁMICA DE LA VIDA URBANA ${ }^{1,2}$
}

MENTAL HEALTH, URBANISM AND CITIES: 1. ON THE PSYCHOSOMATICS AND PSYCHODYNAMICS OF URBAN LIFE

\section{RESUMEN}

El trabajo se realizó a partir de una invitación de las entidades vecinales de La Mina, un barrio de la conurbación de Barcelona cargado de factores de riesgo psicosociales y sociales. La propuesta del trabajo consiste en reflexionar sobre el futuro del propio barrio desde la perspectiva de la salud mental teniendo en cuenta las actuaciones urbanísticas que se están realizando a su alrededor, tales como el «Forum de las Culturas» y la nueva reordenación del litoral barcelonés.

Se recogen experiencias y estudios de las Unidades de Salud Mental (adultos, niños y drogodependencias) que durante 18 años actuaron en dicho medio. Se hace hincapié en la importancia de la «red profana» de contención y en las dificultades de la intervención profesionalizada

\section{ABSTRACT}

The work was carried out starting from an invitation of the local entities of The Mine, a neighborhood of Barcelona's conurbation loaded with many social and psychosocial risk factors. The proposal of the work consists on thinking about the future of the own neighborhood from the perspective of the mental health, keeping in mind the urban plans and changes that are being carried out to its surroundings, such as the Forum of the Cultures and the new reordering of the Barcelona seaside.

We begin from our experiences and studies of the Mental Health Units of La Mina (adults, children and drugs unit) during 18 years. Stress is made in the importance of the "profane network of contention» and in the difficulties of

1. Redacción aproximada de la conferencia-coloquio realizada dentro de la XIII Setmana Cultural de La Mina, organizada por el Centro de Atención Primaria La Mina y la Plataforma d’Entitats i Veïns del Barri de La Mina. (Sant Adriá del Besós 20 de mayo del 2004).

2. La realización de este artículo está apoyada en el amplio trabajo bibliográfico, muestral y de campo realizado en el Proyecto SASPE (Señales de Alerta y Signos Prodrómicos de la Esquizofrenia) y el Proyecto LISMEP (Listado de Items de Salud Mental Pre-escolar). El Proyecto SASPE ha recibido una ayuda a la investigación (02E/99) de la Fundació Seny, tras evaluación a cargo de la Agencia para la Evaluación de la Investigación y las Tecnologías Médicas de Catalunya. El Proyecto LISMEP ha sido apoyado por la Ayuda TV02/ 0133 de la Fundación MaratóTV3, tras la evaluación a cargo de la Agencia Nacional Española de Evaluación y Prospectiva).

3. Para las concreciones urbanísticas y de planificación he utilizado las reflexiones realizadas conjuntamente con Adriana Dal Cin (+) y Javier de Mesones, doctores arquitectos, especialistas en Planificación. 
en medios urbanos con acumulación de factores de riesgo psicosociales. Esas experiencias y reflexiones se ponen en relación con experiencias claves en salud pública contemporánea, tales como los estudios de Roseto (Pensilvania, USA) y Whitehall (UK). Esa reflexión conflictiva es la que da lugar a un esquema, el de los «niveles para la contención», que desde entonces he utilizado en diversos campos $y$, en especial, para la relación entre planificación regional y salud mental.

Palabras Clave: Factores de riesgo - Factores de riego psicosociales - Psicosomática - Red social - Psiquiatría Social - Salud Mental Comunitaria. the profesionalized intervention in urban zones with accumulation of psychosocial risk factors. Those experiences and reflections are connected with key experiences in contemporary public health, such as the studies of Roseto (Pennsylvania, EUA) and Whitehall (UK). That conflicting reflection is the one that gives place to my model of the «levels for the contention» that I have used from then in diverse fields and, especially, for the relationship between regional planning and mental health.

Key Words: Risk factors - Psychosocial Risk factors - Psychosomatics - Social network - Social Psychiatry - Community Mental Health.

\section{INTRODUCCIÓN}

Para poder realizar esta serie de reflexiones con ustedes creo que he de partir declarándome en buena medida lego en estos temas, pero, por otra parte, aficionado a ellos. Sólo con cierta dosis de atrevimiento, y motivado por la solicitud de las entidades vecinales de La Mina (un barrio de Sant Adriá del Besós, Barcelona, Europa), me atrevo a volver a escribir sobre estos asuntos, después de años de silencio. Bien es verdad que ahora me empuja a hacerlo una nueva motivación: la gran operación urbanística del «Forum» de Barcelona, realizada justo en las fronteras del barrio de La Mina, en el que trabajo profesionalmente hace veinte años y con cuyos vecinos he participado desde hace más de treinta en diversas inquietudes como ciudadano (1).

Y ya que la motivación es tan próxima y vivencial, he creído que podría ser oportuno que para estas reflexiones partiese de tres momentos de mi vida profesional que poseen raíces y repercusiones técnicas propias del urbanismo y la planificación urbanística, campos técnicos bastante alejados de la psiquiatría y el psicoanálisis, que son los míos propios. Lo hago así porque creo que participar en esas vivencias es una forma como otra de entrar en tema. También, desde luego, porque espero que nos puedan servir para reflexionar en común.

Partiré pues de tres momentos de los tres grupos de ciencias y técnicas que estoy intentando relacionar: la salud pública, la salud mental comunitaria y el urbanismo o planificación regional. 


\section{En las montañas de Pensilvania}

El primer momento lo centraré en Roseto, un pueblecito de las montañas de Pocono, en Pensilvania, USA. Pocono y sus alrededores, en los años ochenta del siglo XIX, fueron «colonizados» por inmigrantes muy homogéneos. Todos ellos provenían de la Italia meridional. Vinieron en oleadas, se instalaron en oleadas en la rica tierra de acogida y siguieron viviendo como en familia y como en su pueblo durante decenios. Pero hete aquí que, en los años cincuenta del siglo XX, curiosos epidemiólogos e investigadores sociales descubrieron que en Pocono y sus alrededores las enfermedades cardiovasculares eran menos frecuentes que en el resto de los USA. Significativamente menos frecuentes y graves que en los USA y en gran parte del mundo industrializado. Una legión de estudiosos de salud pública, medicina interna, epidemiología, sociología, antropología cultural, etc. cayó sobre el pueblo y realizó sobre él y sus habitantes estudios que han durado más de 50 años (2-7) En definitiva, toda aquella cohorte de científicos deseaban averiguar qué factores protectores existían en la cultura, costumbres sociales, alimentación, género de vida y hábitos de sus habitantes para obtener esos saludables datos. Y lo que encontraron, al menos durante años, no dejó de llevarles de sorpresa en sorpresa, hasta el extremo que a varios de los más conocidos de entre ellos, como el profesor Wilkinson (2) o el sociólogo Stewart Wolf (8), iba a cambiarles la perspectiva de su especialidad y de su vida (3).

$Y$ es que, para su sorpresa, los sesudos investigadores de salud pública, que pensaban encontrar un pueblo o una ciudad en la cual, por algún motivo desconocido, sus habitantes estuvieran menos expuestos a los conocidos factores de riesgo cardiovasculares (sedentarismo, dieta rica en grasas animales, tabaco, alcohol...), encontraron posiblemente lo contrario: los habitantes de Pocono persistían anclados en sus costumbres tradicionales, propias de la Italia meridional de la que, en su inmensa mayoría, provenían. Ello implica, por ejemplo, suaves y deslizantes lasañas y sabrosas y tiernas albóndigas... En la composición de ambas, la combinación de dos o tres tipos de carne es básica: y entre ellas, la carne de cerdo, fundamental también en sus sabrosas salchichas. Como son básicas las grasas en las salsas de tomate, marinara, pesto, con las que acompañan sus platos y sus guisos. Todo ello regado con vinos italianos, ricos en alcohol, consumidos en generosa cantidad $y$, a menudo, sobre todo tras las copiosas comidas en familia de sábados y domingos, acompañados de cigarros y pitillos. Y alguna grappa u otros destilados similares.

¿Cómo, con esa acumulación de factores de riesgo más que demostrados, los habitantes de Roseto podían poseer esas tasas tan bajas de enfermedades cardiovasculares, la mitad en ocasiones que las del resto del país? Busca que te busca, se llegó a la conclusión de que se necesitaban sociólogos y psicólogos sociales para explicarlo: En realidad, Pocono estaba habitada por campesinos italianos que habían llevado hasta el remoto Nuevo Mundo una rica y equilibrada cultura campesina caracterizada por la sencillez y naturalidad en la alimentación, pero también por las 
relaciones de buena vecindad. Fue Wolf el primero que comenzó a insistir en que el secreto de la salud cardiovascular de Roseto radicaba en la calidad de su vida social $(3,8)$. En efecto, Roseto era, en cierta forma, una comunidad cerrada y bastante autosuficiente: en la mayoría de las casas, como en su Italia de origen, convivían varias generaciones, protegiéndose los unos a los otros de infortunios como la enfermedad, el paro, la marginación... La criminalidad era baja y casi no había ni candidatos a los servicios sociales -algo, por cierto, muy diferente de lo que ocurre en nuestros barrios marginales de la opulenta Europa del Siglo XXI. Todos se conocían entre sí, se saludaban y hablan por la calle, se juntaban los fines de semana en coros, bandas, bailes, plazas, comidas, meriendas... Cuando llegaban a adultos, se apuntaban a clubes italo-americanos, frecuentes y concurridos, donde los hombres pasaban las tardes charlando y jugando a las cartas.

Como describe Helen Epstein en su excelente y sensible trabajo de 1999 sobre el tema (3), aunque en Pocono también había ricos y menos ricos, la exhibición de riquezas era excepcional... Todo el mundo vestía casi las mismas ropas, confeccionadas por los mismos sastres, y vivía en similares casas cuadradas revestidas de madera, con sus porches de entrada y sus clásicas telas metálicas...

Empero, en los años setenta, la vida en Roseto cambió de forma progresiva: los jóvenes habían crecido y se habían ido integrando en el «estilo de vida americano». Las familias se dispersaron cuando los hijos marcharon. La prosperidad económica del país era notable, o sea que la gente de Roseto comenzó construir casas más y más grandes, más y más lujosas... Comenzaron a aparecer diferencias en la edificación, entre unas y otras zonas del pueblo, en los clubes, en las fiestas de fin de semana.... Hacia 1985, la tasa de trastornos cardiovasculares agudos era ya similar a la de las ciudades cercanas. $Y$, sin embargo, en Roseto se consumía menos tabaco que veinte y treinta años antes y los hábitos alimentarios eran más saludables. ¿Qué es lo que producía esa aparente paradoja?

Para Stewart Wolf (8), que pasó casi cincuenta años estudiando Roseto, lo único que había cambiado era el grado de cohesión social: Ahora Roseto era un pueblo grande «como los demás». Había menos meriendas, menos fiestas públicas, menos picnics, menos afiliados a bandas de música, coros y clubes... Algunos de los clubes italo-americanos habían tenido que cerrar incluso. Ya existían desempleados y una lista de espera en los servicios sociales. En definitiva, como en los mismos años estaba mostrando el profesor Richard Wilkinson con su estudio de los funcionarios del Gobierno británico que trabajan encerrados en esos hermosos edificios góticos junto al Támesis (Whitehall), las desigualdades sociales son uno de los más graves factores de riesgo para numerosas enfermedades, y en especial, para las enfermedades cardiovasculares $(2,9)$. Particularmente, si esas desigualdades son vividas con la desesperanza de que nada ni nadie será capaz de superarlas, cambiarlas o saltarlas... En definitiva, si se ha perdido el control sobre la propia vida y la esperanza de recu- 
perarlo. La igualdad económica es importante para la salud, como se había mostrado asimismo en la Inglaterra de la guerra mundial y la inmediata posguerra. Pero Roseto y Whitehall concretaban aún más el fenómeno: una red social activa, una conciencia de solidaridad y camaradería, unas metas sociales compartidas por las cuales luchar, pueden tener más trascendencia para la salud pública que legiones de médicos, sanitaristas y planes sanitarios $(5,9,3)$. Me gusta resumir el tema parafraseando a Balint, cuando afirmaba, con toda razón, que el médico era el principal fármaco de la medicina, incluso moderna $(10,11)$. En el mismo sentido suelo afirmar que la medicina de la solidaridad podría ser la medicina más barata y potente de la sanidad y la farmacopea contemporáneas.

\section{En las suaves colinas escocesas: La Mina y la familia real británica}

La segunda serie de vivencias sobre el tema es personal y ocurre en Glasgow al final del siglo XX. En concreto, en 1993. Se reunía allí aquel año el Congreso Internacional de la ISOCARP, una asociación internacional de urbanistas y «planificadores» regionales». Estaban presentes, por ejemplo, los técnicos que habían intentado la planificación de Lahore, de México DF, de la Comunidad de Madrid, de Arganda, la reconversión del propio Glasgow... Por una serie de razones que nunca he llegado a saber, la dirección del Congreso se puso en contacto conmigo porque uno de los asuntos que habían decidido tratar era el de «Urbanismo y Salud Mental» y querían que dictara una conferencia con claves sobre el tema y que participara en un «trabajo práctico» sobre el asunto. Evidentemente, yo no era la persona más indicada para ese trabajo, por lo que me vi obligado a repasar literatura y a organizar mis escasos conocimientos sobre dicho tema con la ayuda de dos urbanistas y planificadores de reconocido prestigio internacional, una de los cuales hoy ya ha fallecido ${ }^{4}$.

El Congreso fue sumamente interesante, máxime porque se celebraba en una ciudad sometida a una profunda remodelación: Glasgow había sido y vivido como ciudad industrial y obrera, hundida en la oscuridad, las humedades, la niebla, la contaminación y las miserias -como parte de sus edificios góticos y neogóticos parecían hundirse en sus verdes campiñas, tersas e igualadas como británicas moquetas-. Pero en esos años, la ciudad se estaba viendo obligada a trasformarse radicalmente de cara al siglo XXI. Algo que, desde parecidas circunstancias previas, se estaba planteando el gran Bilbao. Algo que, supuestamente, se había intentado ya en cinco ocasiones en el caso de La Mina, Sant Adriá de Besós (Barcelona, España). Al menos experiencias de fracasos sí teníamos: o sea que propusimos un esquema para tener en cuenta los niveles para la contención y protección de la salud mental en el ámbito urbanístico estudiando previamente las dificultades y problemas con los cuales los vecinos y los técnicos se encontraban para trasformar urbanística y funcionalmente un barrio como «La Mina». El trabajo «de campo» y la conferencia fueran ampliamente valorados 
y así, gracias a La Mina, me vi premiado por sorpresa. El premio me fue entregado por un miembro de la familia real inglesa en una cena old style en el Ayuntamiento de Glasgow. No entiendo mucho de monarquía y no sé el grado de parentesco con la reina de Inglaterra de aquél atildado lord, por supuesto ataviado con el clásico kilt en la cena de gala, pero siempre me ha hecho mucha gracia pensar que, al menos por un momento y en un lejano punto de la geografía, La Mina y la familia real británica cruzaron sus trayectorias...

Desde el punto de vista profesional, aquél esquema, generado bajo presiones, me ha sido sumamente útil más adelante: en particular, en todos mis trabajos y colaboraciones sobre promoción y prevención de la salud, muchos de ellas realizadas con compañeros del CAP La Mina (12 - 15) En definitiva, con mi esquema, trato de resumir los niveles o factores que pueden convertirse en factores de riesgo si están alterados, pero que pueden atenderse y cuidarse también como factores de protección y resiliencia para la descompensación mental o emocional, para la psicopatología.

En epidemiología se llama factores de riesgo a las características, variables o contingencias que, si se dan en un individuo o población, hacen que el mismo tenga mayores probabilidades de desarrollar un trastorno que la población general. Los factores de protección son los que logran que un individuo tenga menor probabilidad de padecer ese trastorno que la población general. Los factores de contención o resiliencia $(16$ - 20) son aquellos que, a pesar de la existencia de factores de riesgo, parecen proteger al individuo de la descompensación o el trastorno. La contención sería «esa función o capacidad para percibir y recibir las ansiedades -propias o ajenas-, incorporarlas en nuestro interior, y devolverlas (o no) a un nivel de «metabolización» o elaboración que favorezca más el desarrollo que las ansiedades iniciales inmodificadas» $(19,21)$ : una capacidad de contención aumentada en el individuo, en sus allegados o en su red social significa una protección importante contra el desequilibrio mental en cualquiera de sus formas. El concepto, mucho más reciente, de resiliencia, guarda estrechas relaciones con el de contención: Se suele entender por resiliencia la capacidad de adaptarse y soportar las situaciones y factores de riesgo desfavorables sin caer en la enfermedad, desorganización o la psicopatología, fundamentalmente mediante las capacidades individuales o grupales de adaptación y flexibilización ante los envites de tales contingencias $(12,16)$

Pero no hay que olvidar que los factores de riesgo no son estáticos. Cambian en relación con la fase del desarrollo del individuo, los conflictos, estresores y pérdidas afectivas de la vida, la diversidad de dispositivos de la red social o familiar... Algunos factores de riesgo, tales como los derivados de la familia y el género parecen ser fijos, al menos en la vida adulta, pues forman parte de la dotación biopsicológica del individuo y no son modificables «hacia atrás». Empero, no hay que olvidar que sí es maleable la «representación mental» de los mismos (uno de los objetivos de la psicoterapia psicoanalítica) y, desde luego, aún son maleables y modificables en la 
edad infantil. Otros factores de riesgo, tales como la falta de apoyo o red social, las dificultades culturales, diversos tipos de exposiciones a tensiones innecesarias, etc. pueden ser mejoradas mediante intervenciones adecuadas y planificadas (15). Incluso en los trastornos con más carga hereditaria, como es el caso de la esquizofrenia, los estudios de concordancia muestran que cerca de la mitad de los gemelos monocigóticos no desarrollan el trastorno $(22,23)$ : Ello sugiere la posibilidad, apoyada por la clínica y por estudios poblacionales de que, adecuadamente cuidados esos niños y sus familias desde la infancia, el riesgo de desarrollar el trastorno disminuye.

Por tanto, los distintos tipos de prevención deben tener en cuenta no tan sólo los factores de riesgo, sino también los de protección o resiliencia. Los factores de riesgo generales o inespecíficos son los que resultan comunes en muchos trastornos mentales. Por ejemplo, a nivel individual, los déficits neurofisiológicos, las dificultades temperamentales, las enfermedades físicas crónicas, la inteligencia por debajo de la media.... Otros factores de riesgo inespecíficos son familiares: desavenencias conyugales graves, minusvalía o marginación social, hacinamiento, criminalidad o uso de drogas en los padres, trastorno mental materno, institucionalización en la infancia... $(17,18,24)$. Asimismo hay que tener en cuenta los factores comunitarios, tales como la marginación o disgregación social, la escolaridad inadecuada o interrumpida, las catástrofes sociales, la falta de solidaridad social, las situaciones sociales que son vividas con incapacidad de cambiarlas, etc. (1, 2-9, 25 - 35).

También hay que recordar que existen factores que determinan una vulnerabilidad sobre la cual los factores de riesgo pueden facilitar aún más las evoluciones desfavorables. Por ejemplo, el paro y el desempleo no deseados o la marginación social predisponen a determinadas enfermedades y a conflictos psicosociales y trastornos psicológicos, bien estudiados desde hace decenios $(17,18)$. También la acumulación de factores de riesgo puede aumentar la tendencia a que aparezcan trastornos, bien en el futuro inmediato o en la infancia, bien años después o en la edad adulta (36).

\section{CONCRETANDO LOS TEMAS EPIDEMIOLÓGICOS Y PREVENTIVOS CON LA EXPERIENCIA DE LA MINA}

Y hablando de acumulación de factores de riesgo, es el momento de hablar de La Mina, en Sant Adriá del Besós (Barcelona). Se trata de un barrio que se creó «ex novo» para eliminar otros barrios o focos de marginalidad y chabolismo. El resultado es uno de las zonas de la conurbación de Barcelona en las cuales coinciden más crudamente unos objetivos urbanísticos como poco equivocados y una realidad social ya problemática de origen, que se ha ido degradando con el paso del tiempo y que continúa en proceso de degradación. Un barrio, por tanto, en el que puede suponerse ya de entrada una alta incidencia y prevalencia de trastornos mentales y trastornos «psicosomáticos» (es decir, enfermedades muy influidas por factores de 
riesgo psicológicos y sociales). Pero, sobre todo, un barrio en el cual los conflictos psicológicos y psicosociales, así como los trastornos mentales, corren el riesgo de cronificarse por la acumulación de factores de riesgo biológicos, psicológicos y sociales. Un barrio en el cual, posiblemente, el trabajo en el ámbito de la salud mental se hallará especialmente dificultado y el trastorno mental, mucho más omnipresente y crónico. Tanto que, entrando ya en la tercera serie de experiencias que me han movido a estas reflexiones, es habitual y casi sistemático que los estudiosos de turno tiendan a olvidar que existen dispositivos que intentan cuidar de los trastornos mentales y la salud mental del barrio. Es como si pensaran que se notan tanto los trastornos que «aquí no debe haber nadie que los atienda». En el fondo, eso implica una perspectiva profundamente idealista de lo que puede hacerse en nuestro campo de trabajo $-\mathrm{y}$, en ocasiones, una perspectiva profundamente sectaria de en qué consiste la salud (mental). Pero las presiones en esa dirección son tan llamativas que incluso en los estudios previos a la redacción del último Plan de Transformación del barrio de La Mina (2000-2010), el Plan de Reordenación y Mejora del barrio de la MINA (PERI), se afirmaba que no había dispositivos para atender a la salud mental del barrio. Hasta que esos «estudios» llegaron a nuestras manos, claro. Y es exactamente al contrario: con la pobreza e insuficiencias que sea, pero llevamos cerca de veinte años atendiéndola. Sólo que, tal vez, los trastornos mentales y por adicciones de sus habitantes son más visibles que en otros barrios. También más numerosos, sí. Pero sobre todo, más visibles en los «eventos consuetudinarios que ocurren en la calle»; desde luego, más comentados por la prensa.

Es difícil mantener que no se atiende a los problemas de salud mental del barrio cuando han pasado por su Centro de Salud Mental de Adultos y su Centro de Salud Mental Infanto-Juvenil más del 25 por ciento de la población de ese barrio. Pero el problema está en qué significa «atender a la salud mental de esos ciudadanos» 0 , dicho en otros términos más técnicos, qué indicadores de salud y trastorno mental son aplicables en esos medios sociales. Si la mayoría de los 5.285 consultantes que nos han visitado a lo largo de estos años estuvieran ingiriendo diariamente psicofármacos, y visitándose en el Centro de Salud semanalmente por ello ¿serían mejores sus indicadores de salud? La asistencia sería mucho más cara y masiva, desde luego, pero ¿su salud sería mejor? Nuestra propia experiencia clínica con esa población, así como los estudios internacionales realizados en barrios oprimidos y en países de la periferia de la riqueza mundial, hacen dudar de esa hipótesis $(20,24)$.

$Y$ es que este barrio posee características que lo asemejan con esos otros barrios y poblaciones de la periferia del sistema. Para bien (tiene una cierta idiosincrasia y red social propia) y para mal (posee la típica acumulación de factores de riesgo social y psicosocial). Para comprenderlo, basta pasear un día cualquiera por sus calles y, a ser posible, un sábado o un domingo. 
Por ejemplo, aún pueden verse cotidianamente por sus calles grupos de madres con sus niños, grupos incluso de madres lactantes, y, por supuesto, mujeres dando el pecho a sus hijos. $Y$ animados corrillos en los que, mientras una mujer da de mamar a su hijo (a veces a más de uno), conversa con otros grupos de co-madres. He ahí una hermosa vivencia de factores protectores actuando y viviéndose en las calles de La Mina. ¡Lástima que algunos de los nuevos planificadores no tengan en cuenta ese dato para pensar y distribuir el «mobiliario urbano!»: Tal vez no es adecuado que, en barrios como éste, cuando se reforma el «mobiliario urbano», se haga plantando bancos unipersonales o bancos colocados lateralmente unos con respecto a los otros, con las dificultades arquitectónicas para la comunicación que eso supone. Bien es verdad que el barrio puede suplir con creatividad esas barreras que «los pensadores externos» a veces imponen: Así como hubo unos años en los que las conexiones ilegales a la red incluso de gas se contaban por centenares, los sufridos pobladores de La Mina ya saben lo que suponen los asientos rígidos $y$, si es necesario, se bajan de su casa sillas de enea o plegables para poder hablar más cómodamente con las co-madres.

Porque una ventaja de la permanencia de las redes sociales estrechas es la confianza con la que pueden expresarse, casi en público, las relaciones y formas de vida de sus moradores. Una expresión del tema que aún me llama la atención es cómo numerosas mujeres de La Mina siguen bajando a la calle ataviadas con sus tradicionales batas caseras de «boatiné», acompañadas incluso en ocasiones por su no menos tradicionales e inseparables compañeras: las zapatillas caseras tradicionales, esas que suelen ir adornadas por una sedosa y ondulante borla en el empeine. Como suelo responder cuando me lo preguntan, creo que el significado concreto de esa costumbre sólo puede otearse en algunas personas concretas, valorando sus características de personalidad y su contexto mediante el pensamiento clínico, la aproximación científica a los seres individuales (24). Pero con un pensamiento clínico ilustrado al menos por la antropología cultural y la sociología: Uno puede salir a la calle en bata y zapatillas, en ocasiones incluso en ropa interior o con rulos en el cabello, porque la distancia entre el interior y el exterior de los hogares aún no es tan abismal y omnipotente como en las típicas ciudades extensas norteuropeas o norteamericanas... Pero puede que salga así por pura desidia, desinterés o incapacidad para arreglarse, es decir, por la des-esperanza social y psicosocial y, tal vez, por la depresión y otros trastornos emocionales crónicos (37-39).

En cuanto al cuidado del cuerpo y al uso del mismo para cuidar la salud mental, es cierto que, como me decían en una tienda, «ahora por el barrio se ve mucha gente en chándal, corriendo o haciendo como que corre. Incluso señores y señoras mayores, de mediana edad». Cierto: El tipo de urbanismo y, otra vez, la tendencia cultural a relacionarse con el vecino, más que a juzgarlo y a aislarse de él, lo favorecen. Pero muchas de las personas que han realizado esa observación no saben que es un fenómeno que, en cierta medida, tiene que ver con la presencia en el barrio de una 
Unidad de Salud Mental fuertemente integrada en el mismo y con alta accesibilidad. En efecto: Por factores que no hemos podido investigar en profundidad, pero vinculados probablemente a los trastornos en las primeras relaciones padres-hijos, las relaciones primigenias, en este barrio hay una alta incidencia de un trastorno mental concreto: La agorafobia y diversos tipos de crisis de angustia. $Y$ en los medios técnicos de la salud mental, para ese tipo de trastorno suelen recomendarse tres tipos de tratamientos: el uso de psicofármacos (antidepresivos y /o ansiolíticos) durante años, la realización de psicoterapias o diversos protocolos mixtos.

Personalmente, no conozco ningún estudio, ninguna demostración de que, a medio plazo, los tratamientos basados únicamente en medicación (ansiolíticos y /o antidepresivos) proporcionen mejorías duraderas. A menudo proporcionan mejorías a corto plazo, pero pocos estudios médicos o psicológicos se han ocupado de ver qué ocurre en el ámbito biológico, psicológico y social con las personas que ingieren esos fármacos durante años o incluso se hacen dependientes de los mismos. Excepto para tres franjas de edad: infancia, adolescencia y ancianidad. Hoy ya sabemos que, en las dos primeras, deben evitarse, como ya veníamos manteniendo hace años $(19,40,41)$. En la última, en la ancianidad, que deben monitorizarse con sumo cuidado. $Y$ en las edades intermedias, ni la clínica ni los pocos estudios con cierta duración realizados apoyan ese uso crónico de fármacos para ese trastorno. Si existen estudios, al contrario, que apoyan el uso de psicoterapias y, sobre todo, de terapias combinadas (con componentes biológicos, psicológicos y psicosociales) para los mismos (42). Hace años que, junto con la población a la cual atendemos, venimos buscando vías para atender a esos y otros trastornos. Pero nuestra visión de las terapias en salud mental nos impulsa a tener siempre en cuenta una perspectiva amplia de los medios terapéuticos con los cuales una sociedad subviene a las necesidades de sus miembros. En consecuencia, nuestra perspectiva de las posibilidades terapéuticas tendrá en cuenta no sólo las medicaciones y otras medidas biológicas, sino, también, las medidas psicológicas, las psicosociales $\mathrm{y}$, por supuesto, las redes sociales. Es nuestro esquema, en el que llevamos trabajando hace años, de los «niveles para la contención y resiliencia» (Tabla 1 ). Mantenemos que, tanto en el caso de conflictos, como de duelos, como de trastornos mentales, hay que tener en cuenta esos niveles para la contención y escoger en cada caso y situación el fundamental (43). Pero el fundamental en función de su eficacia y efectividad en el cuidado del problema a tratar teniendo en cuenta las características y contexto de la persona 0 grupo a la cual queremos ayudar. Es lo que he llamado la atención sanitaria centrada en el consultante en tanto que miembro de la comunidad (13,19). Por eso, nuestra guía clínica para el tratamiento de la agorafobia y las crisis de angustia no consiste únicamente en fármacos, sino en un tratamiento combinado en el cual incluimos en lugar destacado la integración o re-integración en la red social, las ayudas psicológicas -ya que, por nuestra pobreza de personal, no podemos realizar sino contadas 
psicoterapias-y los «sistemas profanos y para-profesionales» (19). Y ahí la práctica del deporte, mejor en grupo que individualmente, juega un papel descollante: El deporte, el ejercicio físico, puede jugar un importante papel en la socialización, genera secreciones internas que son a la vez ansiolíticas y antiálgicas, proporciona mejorías en la autoestima y en la capacidad de autocontención, cambia la dinámica mental, la dinámica de nuestros pensamientos y sentimientos, promueve la vida social... En resumen: Es una recomendación que proporcionamos para ese tipo de trastorno. $Y$ tanto de forma continuada, es decir, en el ámbito de prevención primaria, como en el ámbito de prevención secundaria, en el ámbito terapéutico: ayudamos a nuestros pacientes a identificar los primeros signos de las crisis $y$, si les es posible, a poner en práctica medios primero sociales -compañía y relación--, y, si no basta, ejercicio físico para intentar contener esa situación puntual de angustia puntual. Un buen sistema es lanzarse a realizar, en esos momentos, una cierto esfuerzo físico. $Y$ ahora entienden ustedes esa proliferación en el barrio de personas de cualquier edad y género que corren y corren: ${ }_{i} A$ correr!

Tabla 1

Los niveles para la contención de las presiones hacia el desequilibrio (mental)

\begin{tabular}{|l|}
\hline 1. El mundo interno («objetos internos») \\
\hline 2. Las capacidades yoicas, personales (para conducirnos con nuestros impulsos y con la sociedad) \\
\hline 3. El cuerpo y la representación mental del cuerpo \\
\hline 4. La familia real \\
\hline 5. Las redes profanas o red social \\
\hline 6. El trabajo y las relaciones alrededor del trabajo.. \\
\hline 7. Las redes profesionalizadas: pedagógica, sanitaria, de asistencia social... \\
\hline
\end{tabular}

Ítem más: ¿Están ustedes seguros de que las redes de dispositivos psiquiátricos poseen mejores sistemas de cuidados de la salud mental que la red «profana» de ornitólogos aficionados que en determinados barrios de Barcelona con amplio componente de origen andaluz constituyen espontáneas redes «profanas» para el cuidado y exhibición de sus «canarios»? Esas personas, mayoritariamente hombres, pasan las mañanas de sábados o domingos «paseando sus canarios» (realmente, no tan sólo a nivel simbólico), hablando de ellos, cuidándolos solícitamente en las plazas públicas soleadas de La Mina, l'Hospitalet de Llobregat, Sant Adriá del Besós, Santa Coloma, San Cosme... Tal vez mientras tanto tomen «alguna cervecita que otra» pero ¿saben ustedes de alguna forma de socialización más inocua para algunas de las personas que todos conocemos en esos grupos? Porque a esas actividades también van y pueden ir los agorafóbicos (¿un canario es suficiente objeto contrafóbico?), los pacientes 
con trastornos de personalidad límite, las personas con dificultades intelectuales y con ciertas minusvalías, los pacientes psicóticos, especialmente jóvenes, los adictos a drogas, algunos pacientes sociópatas... ¿Tenemos alternativas mucho mejores para sus «redes sociales»? Y les pongo aquí tan sólo un ejemplo de actividades particulares de nuestros barrios que, sin embargo, deben ser vistas, creo yo, como fuentes de relación, contactos, y mayoritariamente, de salud.

Claro que esa confianza y transparencia para la exhibición de las propias cualidades (los canarios y jilgueros) y defectos (las crisis de angustia) pueden darse también de forma extrema en nuestros barrios y en ese caso no son indicativas de salud ni de salud (mental). Como ejemplo inolvidable recuerdo a un adolescente gravemente afectado por conflictos emocionales que vivió durante semanas en una tienda de campaña en el campo de fútbol del barrio, cuando su padre le echó violentamente de casa...

$Y$, alternativamente a esa realidad, la tendencia a la disociación de los trastornos mentales y a la visión idealizada y omnipotente de los cuidados de los mismos, pueden llevar a la situación ya descrita en la cual los sesudos sociólogos realizan su informe previo... afirmando que no existen dispositivos para esos temas en el barrio. Aún hoy me sigo preguntando si lo hicieron por despiste o porque el sufrimiento mental y psicosocial es tan omnipresente y visible en las calles de estos barrios metropolitanos que debieron pensar que nadie lo cuidaba.

Pero ese error garrafal y frecuente, sintomático, tal vez basado en los valores de omnipotencia y magia que concedemos a lo médico, y, de rebote, a lo psicológico y psiquiátrico (siempre hay un psicólogo o, mejor aún, un medicamento «que lo va a resolver todo»), llegó al último extremo con ocasión de los planes de remodelación del Centro de Salud de La Mina. El Centro de Salud de La Mina es uno de los más antiguos del estado, pionero y abanderado de la línea de Atención Primaria aún hoy. Desde 1986 cuenta con un equipo de salud mental que, además, de forma también pionera, integraba el cuidado de los pacientes adictos a drogas en su cartera de servicios: entre 1988 y 1998, por más señas. Pues bien, cuando en el 2000 se decidió cambiar las vetustas y anticuadas instalaciones del centro y se realizaron los planos del nuevo edificio... jse olvidaron del Centro de Salud Mental de Adultos y del Centro de Salud Mental Infanto-Juvenil que ya existían en él! Como consecuencia, no existían locales para los mismos en la remodelación. Y como segunda consecuencia: la remodelación quedó vergonzantemente detenida durante años. Y eso no fue un error de una empresa cualquiera contratada para hacer los planos, no. Los planos y la remodelación del CAP La Mina los había decidido y encargado el propia Servei Catalá de la Salut, el propietario y financiador del Centro y de cada uno de sus equipos: es decir, la administración sanitaria. Y se había «olvidado» de que existían allí equipos de salud mental desde hacía más de 14 años, equipos que atendían o habían atendido a más de 5.000 habitantes del barrio... ¿Cómo explicar esa actuación de grupos de expertos, grupos de la administración y técnicos, uno tras otro, comisión tras comi- 
sión y comité tras comité hasta que, cuando enseñaron el proyecto, ya a la primera ojeada el director del Centro y yo mismo pudimos constatar el «olvido», auténtico lapsus freudiano magnificado y «hecho planos»? El propio Freud debe estar aún contrayéndose de risa en su urna griega del Golder's Green londinense... Pero la cuestión no es una broma: el «lapsus freudiano», como todos los lapsus, tienen un origen y unas consecuencias. ¿Repercusiones y consecuencias?: El plan de remodelación debió detenerse, replanteándose espacios y volúmenes. Otra consecuencia: la población y los profesionales, que debían haber contado con un nuevo centro de salud en 2003, a fecha de mayo del 2005 no conocen aún ni los nuevos planos. ¿Motivaciones? Son sólo hipótesis, pero, en cualquier caso, no se puede pensar muy bien de un organismo «planificador y financiador de los servicios sanitarios» que comete tamaño desaguisado - o de sus dirigentes y su modelo organizativo. Problemas y dudas de la planificación, ambivalencias y conflictos de la misma en barrios como el que nos ocupa.

Otros ejemplos que apoyan mi idea de lo seriamente que hay que plantearse la relación entre la planificación urbanística y sus repercusiones en la salud mental de los pobladores de nuestras ciudades, podría ilustrase con algunas otras experiencias, en este caso con la población de dichos barrios. Los barrios de los que venimos hablando suelen conllevar una acumulación en ellos no sólo de factores de riesgo sociales, sino incluso biológicos, psicosociales y psicopatológicos. Es decir, en ellos se acumulan problemas biológicos (Tabla 2), problemas psicosociales (Tablas 3 a 5: 1, 44 - 46) y los problemas psicopatológicos más graves (Tabla 6) $(14,24,47,48)$.

Comencemos por el ejemplo del «mobiliario urbano»: Tal vez la nueva moda barcelonesa de los bancos fijos o rígidos atados al terreno de forma asimétrica, en ocasiones en con bancos individuales, puede ser un entorpecimiento para su uso $y$, sobre todo, para la socialización, más que favorecer la misma. Pero es cierto que bancos no sólidamente anclados al suelo, y en ocasiones incluso éstos, desaparecen de la vía pública para reaparecer en el «salón-comedor» de alguna casa del barrio o en la «torreta» de los fines de semana de alguno de sus moradores. Aunque, a otro nivel, un exponente de la vida y el gracejo de muchos habitantes del barrio es verles los sábados y domingos soleados a las puertas de sus casas, o en los mugrientos jardines y plazas que aún subsisten entre bloques, departiendo amigablemente, sentados en sus propias sillas de enea, mimbre, madera, que bajan y suben de sus casas para la ocasión. La calle con mobiliario público-privado se convierte entonces en lugar de encuentro, charla, escarceos amorosos ocasionales, apuntes de baile incluso... Y, desde luego, en lugar de encuentro de co-madres: es decir, de madres de edad similar. ¿Recuerdan ustedes de los valores preventivos y de promoción de la salud de la lactancia materna? Pues el grupo de co-madres es una de las formas más acreditadas de sostenerla (49). 
Tabla 2

Características y Factores de Riesgo de la población del barrio de «La Mina», en la metrópoli de Barcelona

\begin{tabular}{|c|c|}
\hline Característica & $\begin{array}{c}\text { Estimación sobre la } \\
\text { población total }\end{array}$ \\
\hline Población & $18.657-23.657^{*}$ \\
\hline Índice de embarazos en adolescentes & $\begin{array}{c}>\text { que en población general } \\
\text { pero indeterminado }\end{array}$ \\
\hline Incidencia de psicopatología infanto-juvenil detectada en servicio en un año. & $17 \%$ \\
\hline Incidencia de psicopatología en adultos detectada en servicio en un año & $1{ }^{\prime} 02 \%$ \\
\hline Porcentaje de la población visitada en algún momento por la Unidad de Salud mental & $>20 \%$ \\
\hline $\begin{array}{l}\text { Drogodependencias detectadas } \\
\text { - ADVP } 350 \\
\text { - Metadona } 160 \\
\text { - Otras drogas } 500 \\
\text {. TOTAL } 1.010\end{array}$ & $439 \%$ \\
\hline Personas con SIDA y HIV + detectadas 180 & $00^{\prime} 78 \%$ \\
\hline $\begin{array}{l}\text { Etnias } \\
\text { - Caucásicos } \\
\text { - Etnia gitana: } \\
\text { - Otras etnias }\end{array}$ & $\begin{array}{c}43{ }^{\prime} 5 \% \\
33 \% \\
\text { 0'9 \% }\end{array}$ \\
\hline Analfabetos detectados: 2.562 & $14 ’ 32 \%$ \\
\hline Paro (Datos oficiales de 1996) & $54 ' 64 \%$ \\
\hline Jubilados: 3.964 & $16{ }^{\prime} 75 \%$ \\
\hline Pensionistas e inválidos: 5.407 & $222^{\prime} 85$ \\
\hline $\begin{array}{l}\text { Alto índice de enfermedades crónicas: } \\
\cdot \text { HTA: } 2.620 \\
\cdot \text { Dislipemias: } 2.110 \\
\cdot \text { Diabetes: } 1229 \\
\cdot \text { EPOC: } 950 \\
\text {. Enfermedades reumatológicas: }>1000 \text { casos }\end{array}$ & $>25 \%$ \\
\hline Inmigrantes extracomunitarios: 246 & $\begin{array}{c}13 \text { '18 sobre } \\
\text { la población censada }\end{array}$ \\
\hline Media de Hijos por familia & 2'6 (Barcelona: 1'7) \\
\hline Media de Personas por familia & 5'6 (Barcelona: 3’3) \\
\hline
\end{tabular}

* Población censada / Población estimada al alza utilizada por los administradores sanitarios del sector para incluir no censados y posibilidad de «deriva social».

Tabla 3

Problemáticas atendidas por los Servicios Sociales de La Mina en el 2002

\begin{tabular}{|l|c|}
\hline Problemáticas económico-laborales & 422 \\
\hline Problemáticas de Salud & 254 \\
\hline Dificultades de relación familiar & 196 \\
\hline Dificultades con los aprendizajes & 175 \\
\hline Dificultades de vivienda & 92 \\
\hline Dificultades por inadaptación social & 77 \\
\hline Dificultades por inmigración & 19 \\
\hline
\end{tabular}


Tabla 4

\title{
Características urbanísticas negativas del «Polígono Residencial de La Mina» hasta el 2002: «barraquismo vertical»
}

CARACTER: Zona residencial urbana intensiva de bloques aislados

- Barrio segregado de su municipio mediante:

- río caudaloso y canalizado.

- Vías rápidas, enormemente complejificadas con las construidas a propósito de las Olimpíadas de 1992.

- Vía férrea con varios ramales.

- playa degradada y contaminada

RESULTADO: barriadas sin delimitar ni unir a Barcelona-ciudad.

- Zonas industriales en el interior del barrio o rodeándolo:

- Incineradora, depuradora de aguas residuales, cocheras de autobuses urbanos, central térmica,

fábrica de bidones y otras naves industriales.

Zonas de comunicación y esparcimiento realizadas sin contar con las características idiosincrásicas de la población

--> gran degradación y deterioro de las mismas.

- Edificaciones realizadas con la misma falta de atención a las características idiosincrásicas de la población:

«barrio vertical» para familias barraquistas y gitanos (urbanismo anterior y cultural «horizontal»).

- Ocupación del barrio sin definir o elaborar previamente la agrupación de las familias, ni por etnia, ni por «barrio

de barracas» de procedencia, ni por otro criterio.

- Acceso a los pisos por vías complejas:

- demostrando habitación en alguno de los barrios de barracas de procedencia.

- posteriormente, por vía ilegal, mediante organizaciones marginales. Consecuencias:

. gran número de morosos en el pago de alquileres (subvencionados)

. las viviendas se hallan casi totalmente fuera del mercado inmobiliario: la mayor parte de las vías de acceso,

trueque y traslado de vivienda son ilegales.

\section{TABLA 5 \\ Esquema de los problemas del barrio desde el punto de vista social y psicosocial: inestabilidad}

\begin{abstract}
1. Inestabilidad FAMILIAR: Deterioro importante en las relaciones de pareja y en las relaciones padres-hijos: abandonos o semi-abandonos, separaciones, incapacidad de la madre, el padre 0 ambos para atender a los hijos por causas mentales, pobreza y condiciones económicas extremas, malos tratos, drogodependencia con deterioro familiar grave, alcoholismos, trastornos mentales en los padres ---> desestabilización de los niños y adolescentes.

2. Inestabilidad CULTURAL: Anomia importante en las dos culturas, con las consecutivas actitudes antisolidarias, redes delictivas y de «protección», chauvinismo, aislamiento cultural y social, inhibición ante la promoción y la formación... (en especial, de las mujeres).

3.Inestabilidad ECONÓMICA: Alto índice de paro, actividades económicas marginales o delictivas, numerosos pensionistas y jubilados con pensiones mínimas, impago moroso de alquileres, transacciones ilegales de viviendas, ocupaciones ilegales de viviendas, uso ilegal de las redes públicas de agua, gas y electricidad...

4. Inestabilidad URBANíSTICA: Continuos «planes de remodelación» y continuos rumores de «demolición» del barrio.

5. DELINCUENCIA: Tráfico de drogas, compra-venta de oro y otros objetos, tráfico de armas, «protección» de establecimientos mediante amenazas, ambiente urbano deteriorado, especialmente por la noche...

6. Problemas de la INFANCIA Y LA ADOLESCENCIA: Absentismo escolar (especialmente de las niñas y jóvenes), inadaptación social y cultural, actividades marginales y delincuenciales, internamientos en instituciones por deficiencias familiares, deficiencias mentales y físicas más frecuentes, alteraciones familiares y en la capacidad de cuidado y contención de los padres, condiciones socioeconómicas extremas, abandonos totales o parciales por parte de la madre y/o el padre...
\end{abstract}




\section{TABLA 6}

Los factores sociales influencian incluso en los trastornos mentales más graves: Prevalencia de la esquizofrenia y «otras psicosis» en el barrio de La Verneda y en el de La Mina de la Unidad de Salud Mental de Sant Martí (Barcelona), años 1982-2000.

\begin{tabular}{|c|c|c|c|c|c|c|c|c|c|c|}
\hline & \multicolumn{5}{|c|}{ Población General } & \multicolumn{5}{|c|}{ Población en edad de riesgo } \\
\hline & \multicolumn{2}{|c|}{$\begin{array}{l}\text { La Verneda } \\
\text { (3) }\end{array}$} & \multicolumn{2}{|c|}{$\begin{array}{l}\text { La Mina } \\
\qquad(4)\end{array}$} & \multirow[b]{2}{*}{$\begin{array}{c}\text { Diferencia de } \\
\text { prevalencias } \\
\text { (I.C. 95\%) }\end{array}$} & \multicolumn{2}{|c|}{$\begin{array}{l}\text { La Verneda } \\
\text { (5) }\end{array}$} & \multicolumn{2}{|c|}{$\begin{array}{l}\text { La Mina } \\
(6)\end{array}$} & \multirow[b]{2}{*}{$\begin{array}{l}\text { Diferencia de } \\
\text { Prevalencias } \\
\text { (I.C. 95\% ) }\end{array}$} \\
\hline & N & $\begin{array}{l}\text { Preva- } \\
\text { lencia } x \\
10.000\end{array}$ & N & $\begin{array}{c}\text { Preva- } \\
\text { lencia } \\
X \\
10.000\end{array}$ & & N & $\begin{array}{c}\text { Preva- } \\
\text { lencia } \\
x \\
x \\
10.000\end{array}$ & N & $\begin{array}{l}\text { Preva- } \\
\text { lencia } x \\
10.000\end{array}$ & \\
\hline Esquizofrenia (1) & 296 & 37,01 & 180 & 76 & $39,1(27,2-50,9)$ & 262 & 58,1 & 161 & 116,1 & $58(38,9-77,2)$ \\
\hline Otras Psicosis & 223 & 27,88 & 139 & 58,7 & $30,9(20,5-41,3)$ & 133 & 29,5 & 85 & 61,3 & $31,8(17,9-45,8)$ \\
\hline Trastornos delirantes & 145 & 18,13 & 64 & 27 & $8,9(1,6-16,2)$ & 99 & 21,9 & 45 & 32,4 & $10,5(0,09-20,9)$ \\
\hline $\begin{array}{l}\text { Otras psicosis del } \\
\text { adulto (2) }\end{array}$ & 47 & 5,87 & 46 & 19,4 & $13,6(7,7-19,4)$ & 32 & 7 & 37 & 26,6 & $19,6(10,7-28,5)$ \\
\hline $\begin{array}{l}\text { Otras psicosis de } \\
\text { inicio en la infancia } \\
\text { (0-14 años) (8) y (9) }\end{array}$ & 31 & 33,6 & 29 & 72,6 & $39(10,1-67,8)$ & & & & & \\
\hline Totales & 519 & 64,9 & 319 & 134,8 & $69,9(54,2-85,7)$ & 395 & $\begin{array}{c}87,6 \\
(7)\end{array}$ & 246 & $\begin{array}{c}177,5 \\
(7)\end{array}$ & $89,9(66,3-113)$ \\
\hline
\end{tabular}

(1) incluye los casos de esquizofrenia, los trastornos esquizofreniformes y los trastornos esquizoafectivos.

(2) incluye los trastornos psicóticos breves, los trastornos psicóticos compartidos, los trastornos psicóticos debidos a enfermedad médica, los trastornos psicóticos inducidos por substancias y los trastornos psicóticos no especificados.

(3) Población General La Verneda $=79.958$

(4) Población General La Mina $=23.657$

(5) Población en Edad de Riesgo, 15-54 años, La Verneda = 45.071

(6) Población en Edad de Riesgo, 15-54 años, La Mina = 13.859

(7) Se excluyen los casos de otras psicosis de inicio en la infancia.

(8) Población de 0-14 años, La Verneda $=9.211$

(9) Población de 0-14 años, La Mina = 3.993

Pero no es fácil mantener un espacio abierto para lo público en un mundo en el cual lo público desaparece casi cada día en las formas más cotidianas y anodinas: Por ejemplo, cuando pusimos en la sala de espera pequeñas sillas de vistosos colores para que los niños pudieran sentarse en ellas, una de las tres desapareció en la primera semana. Y en más de una ocasión hemos tenido que parar en la puerta al churumbel correspondiente que se llevaba alguna de las otras dos, posiblemente para casa. 0 para ser vendida en el mercadillo más próximo. Claro que el error de esa 
forma privada de ver lo público se corresponde especularmente con la tendencia a hacer igual de la administración y algunas empresas en un mundo dominado por el dogma de «privatiza, que es mejor».

Esas sillas hacían juego con una mesa que poseían un vacío en el centro del tablero, una mesa de diseño escogida también por el Servei Catalá de la Salut y proporcionada, como casi todo el material de los centros catalanes, por una gran empresa transnacional. En teoría, el vacío u orificio servía para dejar allí las construcciones y juguetes con los cuales los niños podían entretenerse en la sala de espera: Pero los juguetes habían desaparecido a las pocas semanas de comprarlos. Y entonces sólo quedó el agujero... No tuvimos suficiente agilidad mental como para pensar en la necesidad de los niños de buscar y meterse en cavidades y claustrum que los contengan y en que la mesa podía jugar ese papel... Hasta que un niño de pocos años quedó encajado de tal forma en dicho agujero que, para sacarle de allí, hubo que hacer venir al servicio de mantenimiento del centro $y$, más tarde, a la policía y los bomberos. Ni los bomberos pudieron sacarlo buenamente: hasta que uno de ellos «se la jugó» en una maniobra brusca y que podía haber producido lesiones al niño... También en este caso nos faltó la agilidad mental previa para «identificarnos» más con los pobladores de estos barrios $y$, por ejemplo, pensar con la mente de un niño de esos barrios... Y, si para nosotros no había resultado posible a tiempo, es de temer el tipo de pensamiento de la administración. Sin embargo, habría que planificar teniendo en cuenta esas necesidades y placeres de los niños pequeños de reencontrar y explorar el interior de la madre ( $y$, por tanto, el interior de todos los agujeros y espacios contenedores) y de salir triunfantes de la cueva amenazadora en la que a menudo se convierten esos interiores y sus representantes simbólicos: escondites, pozos, agujeros, cavidades, cuevas...

Y por cierto: Hemos hablado de la tendencia a «privatizar lo público». Pero eso es lo que hacen o han hecho las mayores empresas de nuestra sociedad. E incluso hay gobiernos que se han aplicado entusiásticamente al asunto, tomándolo como la prioridad de su mandato. ¿Cómo hacer que en la población se mantenga entonces una ética de lo público, lo común, la solidaridad...? Durante un tiempo intenté que en cada una de nuestras salas de espera se pusiera un cartel que rezara algo así como: «La propiedad pública es de todos. Cuídala». El cartel nunca llegó a ponerse: Cada vez que lo decía, no pocos de los miembros del equipo torcían el gesto, no sé si por origen cubano del eslogan (ila dictadura cubana!) o tal vez por el displicente mal cuidado con el que, a menudo, tratamos nuestras propias instalaciones y espacios de trabajo públicos: No siempre lo que falta es pintura. A menudo lo que falta son actitudes elementales de conservación de la estética del propio espacio de trabajo, tan frecuentemente invadido en la sanidad pública por cartones, cajas, folletos, anuncios, mapas, planos, cajones, impresoras, computadoras y otros trastos viejos, utensilios arrinconados y demás «decorados habituales» de nuestros lugares de 
trabajo: ¿Cómo planificar teniendo en cuenta estas tendencias, contradictorias pero tan arraigadas incluso en nosotros, los cuidadores? Es indudable que todo servicio necesita un «cuarto trastero»... También, que, si existe, siempre estará lleno. ¿Cómo resolver el dilema?

Y si a ello le añadimos que el mobiliario urbano (y el de nuestros Centros) desaparece una y otra vez (incluso los bancos públicos), que los campos de deportes a menudo están cerrados a cal y canto porque sus instalaciones no fueron pensadas para la destructividad que van a tener que soportar, que las instalaciones son malbaratadas y averiadas a las pocas semanas de ser inauguradas, si a ello añadimos toda esa serie de experiencias, tal vez la consecuencia es que deberíamos sentirnos obligados a replantearnos nuestros principios no sólo clínicos, sino incluso preventivos y de organización asistencial, cosa que intentamos realizar publicando los protocolos asistenciales que hemos podido ir sedimentando a lo largo de años de trabajo con estas poblaciones (19).

Ampliando la reflexión, lo que nos planteamos es cómo podría ayudar el conocimiento de estos hechos, experiencias y actitudes asistenciales al urbanismo y la planificación y a sus especialistas. En la segunda parte de este trabajo intentaremos proporcionar unas reflexiones y sugerencias al respecto.

\section{BIBLIOGRAFÍA}

1. Dal Cin, A., de Mesones, J., Tizón, JL. La Mina district of Barcelona: A planning approach to psychopathology. Cities 1995;12 (2): 87-95.

2. Wilkinson, RG. Unhealty Societies: The Afflictions of Inequality. London: Routledge; 1997.

3. Epstein, H. Vida y muerte en la escala social. Revista de Occidente 1999; 215(4): 19-47.

4. Marmot, MG. Improvement of social environment to improve health. Geoffrey Rose memorial lecture. Lancet 1998;351(9095):57-60.

5. Marmot, MG. The importance of psychosocial factors in the workplace to the development of disease. En MG. Marmot y RG. Wilkinson (eds.) Social determinants of health. New York: 0xford University Press; 1999 p. 24-38.

6. Marmot, MG. Inequalities in health. New England Journal of Medicine 2001; 345(2), 134-136

7. Marmot, MG. Social class, occupational status and CVD. En Occup Med 2000; 15(1): 22-8

8. Wolf, S., Bruhn, JG. The power of clan. The influence of human relationships on heart disease. New Bruswick, NJ: Transaction Publishers; 1993.

9. Marmot, MG., Wilkinson, RG. (eds). Social determinants of health. New York: Oxford University Press; 1999. 
10. Balint, M. El médico, el paciente y la enfermedad. Buenos Aires: Libros Básicos; 1968.

11. Tizón, JL. Componentes psicologicos de la práctica médica. $6^{\text {a }}$ ed. Barcelona: Biblària;1996.

12. Ciurana, R. Vulnerabilitat i factors de risc a la vida adulta i la vellesa. Llibre de ponències del «II Congrès d'Atenció Primària i Salut Mental». Barcelona, 24 i 25 de Febrer de 2000; 17-25.

13. Tizón, JL. Atendiendo al sufrimiento de los padres: Cuidando el futuro. En Sasot J, Moraga FA (eds). Avances en Psicopediatría: Padres, salud, sociedad. Barcelona: Prous Science 2001. p. 145-67.

14. Tizón, JL. (Coord. y redactor), Salamero, M., Pellegero, N., Sáinz, F., Atxotegi, J., San José, J., Díaz-Munguira, JM. Migraciones y salud mental: Un análisis psicopatológico tomando como punto de partida la inmigración asalariada a Catalunya. Barcelona: PPU; 1993.

15. Tizón, JL., Buitrago, F., Ciurana, R. (Coordinador), Chocrón, L., Fernández, C., GarcíaCampayo, J., Montón, C., Redondo, MJ. Prevención de los trastornos de la salud mental desde la atención primaria de salud. El Programa del PAPPS y la SEMFyC, versión 2003 (con inclusión de los nuevos subprogramas sobre «Violencia doméstica y de pareja» y «Maltratos a los ancianos»). Atención Primaria 2003;32(Supl 2): 77-101.

16. Mangham, C., McGrath, P., Reid, G., Stewart, M. Resiliency: Relevance to Health Promotion. Detailed Analysis. Ottawa: Ministry of Supply and Services; 1995.

17. Institute of Medicine. Risk and protective factors for the onset of mental disorders. Washington DC: Academy Press; 1994.

18. U S Department of Health and Human Services. Mental health: A Report of the Surgeon General. Rockville MD: NIMH; 1999.

19. Tizón, JL., San José, J., Nadal, D. Protocolos y programas elementales para la atención primaria a la salud mental. $2^{\mathrm{a}}$ ed. Barcelona: Herder; 2000.

20. Tizón, JL. Prevención de los trastornos de la salud mental en atención primaria. En C. Brotons, R. Ciurana, M. Iglesias (Editores). Manual de Prevención en Atención Primaria. Programa de Actividades Preventivas y de Promoción de la Salud (PAPPS). Barcelona: Sociedad Española de Medicina de Familia y Comunitaria; 2003 p. 547-575.

21. Tizón, JL. ¿Profesionales «quemados», profesionales «desengañados» o profesionales con trastornos psicopatológicos? Atención Primaria 2004; 33(6):326-30.

22. Alanen, Y. Schizophrenia. Its origins and need-adapted treatment. London: Karnac books; 1997.

23. Tienari, P., Wynne, LC., Moring, J. The Finnish adoptive family study of schizophrenia. Implications for family research. Br.J. Psychiatry. 1994;164: 20-26.

24. Tizón, JL. ¿Se puede pensar todavía en una «sociogénesis» y en una «psicogénesis» de las psicosis? Archivos de Psiquiatría 2004;67(1): 67-72. 
25. Faris REL \& Dunham HW. Mental disorders in urban areas: An echological study of schizophrenia and other psychosis. Chicago: University of Chicago Press; 1939.

26. Hollingshead, AB., Redlich FC. Social class and mental illness. New York: John Wiley; 1958.

27. Bastide, R. Sociologie des maladies mentales. Paris: Flammarion; 1965.

28. Cochrane, R. La creación social de la enfermedad mental. Buenos Aires: Nueva Visión; 1983.

29. Dal Cin, A., de Mesones, JL., Tizón, JL. Marginal Quarters and Mental Health: A proposition of therapeutic planning of «La Mina», Barcelona, Spain. Case Study . En Congress Book of 29th ISOCARP Congress, Plenary Sessions. Glasgow: UNESCO - International Society of City and Regional Planners (ISOCARP)- Association Internationale des Urbanistes (AIU) and Internationale Gesselschaft der Stadt.und Regionalplanner (IGSRP); 1993.

30. Marcelis, M., Navarro Mateu, F., Murray, R., Selten, JP., Van Os, J. Urbanization and psychosis: a study of 1942-1978 birth cohorts in The Netherlands. Psychol Med. 1998; 28,4: 871-79.

31. Salokangas, RK. Living situation social network and outcome in schizophrenia: a five-year prospective follow-up study. Acta Psychiatr Scand. 1997; 96,6:459-68.

32. Santini, S., Caselli, L., Lucareli, S., Trinci, A., Ferrara, M., Zappa, M. Risk factors associated with the phenomenon of long-term care in psychiatry. A case-control study conducted in the Servizio di Igiene Mentale of Cassetino (Arezzo). Epidemiol Prev. 1997; 21,1: 34-40.

33. Tremblay, RE., Phil, RO., Vitaro, F., Dobkin, PL. Predicting early onset of male antisocial behavior from preschool children. Arch Gen Psychiat. 1994; 51: 732738.

34. Torrey, EF., Bowler, AE., Clark, K. Urban birth and residence as risk factors for psychoses: an analysis of 1880 data. Schizophr Res. 1997; 25(3): 169-76.

35. Torrey, EF., Yolken, RH. At issue: is household crowding a risk factor for schizophrenia and bipolar disorder?. Schizophr Bull. 1998; 24,3: 321-24.

36. Amigó, E., Angulo, F., Artemán, A., Callabed, J., Casalé, D., Cerdán, G., Closa, J., Fernández, R., García, E., Ibáñez, M., Prats, R., Puig, M., Sanglás, R., Sasot, J., Tizón, JL., Toro, J., Trías, E. Programa de Seguiment del Nen Sa: Protocol de Salut Mental Infantil i Juvenil. Barcelona: Direcció General de Salut Pública - Departament de Sanitat i Seguretat Social de la Generalitat de Catalunya ;1999.

37. Runciman, WG. Relative Deprivation and Social Injustice: A Study of Attitudes to Social Inequality in Twenty-Century England. LA: University of California Press; 1966.

38. Singh-Manoux, A., Adler N E \& Marmot MG. Subjective social status: Its determinants and its association with measures of ill-health in the Whitehall II study. Social Science and Medicine 2003;56: 1321-33. 
39. Singh-Manoux, A., Britton, A. \& Marmot, M. Vascular disease and cognitive function: Evidence from the Whitehall II Study. Journal of the American Geriatrics Society 2003; 51(10): 1445-50.

40. Puig Antich, J., Goetz, D., Davies, M., Kaplan, T., Davies, S., Ostrow, L., Asnis, L., Twomey, J., Iyengar, S. \& Ryan, ND. A controlled family history study of prepubertal major depressive disorders. Archives Gen Psychiatry. 1989;46:406-41.

41. Jureidini, JN., Doecke, CJ., Mansfield, PR., Haby, MM., Menkes, DB., Tonkin, AL. Efficacy and safety of antidepressants for children and adolescents. BMJ 2004;328:879-83.

42. Tizón, JL. Terapias combinadas en la esquizofrenia: ¿Agregamos, mezclamos y confundimos o bien de-construímos y combinamos? Revista de la Asociación Española de Neuropsiquiatría 2004; 23(90): 97-131.

43. Tizón, JL. Pérdida, pena, duelo: Vivencias, investigación y asistencia. Barcelona: Paidós; 2004.

44. Olin, SC., Mednick, SA. Risk factors of psychosis: identifiyng vulnerable populations premorbidly. Schizophr Bull. 1996; 22 (2): 223-40.

45. Olin, SC., Mednick, SA., Cannon, T., Jacobsen, B., Parnas, J., Schulsinger, F., Schulsinger, $\mathrm{H}$. School teacher ratings predictive of psichiatric autcome 25 years later. Br J Psichiatry. Suppl. 1998; 172 (33): 7-13.

46. Nicolson, R., Lenane, M., Singaracharlu, S., et al. Premorbid Speech and Language Impairments in childhood-Onset Schizophrenia: Association with risk factors. Am J Psychiatry. 2000; 157: 794-800.

47. Jablenski, A. Prevalence and incidence of schizophrenia spectrum disorders: implications for prevention. Australian and New Zealand Journal of Psychiatry. 2000; 34 (Suppl.): 67-79.

48. Ferrer, F. El estado de salud del pueblo Gitano en España. Una revisión de la bibliografía. Gaceta Sanitaria 2003;17:2-9.

49. OMS-UNICEF. Proteccio, promoció y suport de l'alletament matern: La funció especial dels serveis de maternitat. Barcelona: Associació Catalana Pro Alletament Matern; 1992.

Jorge L. Tizón

Neuropsiquiatra, psicólogo, psicoanalista.

Director del Equipo d'Atenció Precoç als Pacients amb Psicosi del Institut Catalá de la Salut de Barcelona 